\title{
Increasing health equity and access to skilled birth delivery services for the poor through community-based health-care interventions: evidence from northern Nigeria
}

\author{
Ekechi Okereke*, Ibrahim Yisa, Adekunle Akerele, Benson Obonyo, Mike Egboh \\ From Health Services Research: Evidence-based practice \\ London, UK. 1-3 July 2014
}

\section{Background}

The Partnership for Transforming Health Systems Phase 2 (PATHS2) project introduced and supports community based interventions such as safe motherhood initiative demand side (SMID) and an emergency transport scheme within northern Nigeria. This study evaluates the effect of PATHS2's community based interventions in addressing transportation as a barrier to accessing skilled birth delivery services in health facilities.

\section{Methods}

The PATHS2 project conducted baseline and midline surveys in 2009 and 2012 respectively to evaluate the impact of its health care interventions. Structured questionnaires were applied to respondents in randomly selected households within PATHS2's intervention communities in Kano and Jigawa States during the baseline and midline surveys. The study respondents were categorized into five wealth quintiles using principal component analysis. Data analysis was undertaken using SPSS version 20.

\section{Results}

Within intervention communities in Kano and Jigawa States, the proportion of respondents in the poorest and second poorest wealth quintiles at baseline relative to midline who indicated that transportation is a barrier to accessing skilled birth delivery services decreased by $28 \%$ and $24 \%(\mathrm{p}<0.001)$ for Kano State as well as $21 \%$ and $25 \%$ $(\mathrm{p}<0.001)$ for Jigawa State respectively. There were no significant decreases in the proportion of respondents

\footnotetext{
Abt Associates Nigeria/Partnership for Transforming Health Systems Phase 2,
} Abuja, Federal Capital Territory, Nigeria 